\title{
Téoros
}

Revue de recherche en tourisme

\section{Prospective du marché du travail en tourisme : du sous-développement à la professionnalisation}

\section{Jean Stafford}

Volume 2, numéro 1, février 1983

Formation et conditions de travail en tourisme

URI : https://id.erudit.org/iderudit/1080840ar

DOI : https://doi.org/10.7202/1080840ar

Aller au sommaire du numéro

Éditeur(s)

Université du Québec à Montréal

ISSN

0712-8657 (imprimé)

1923-2705 (numérique)

Découvrir la revue

Citer cet article

Stafford, J. (1983). Prospective du marché du travail en tourisme : du sous-développement à la professionnalisation. Téoros, 2(1), 20-22.

https://doi.org/10.7202/1080840ar d'utilisation que vous pouvez consulter en ligne. 


\title{
Prospective du marché du travail en tourisme: du sous-développement à la professionnalisation
}

\author{
par Jean Stafford
}

Le marche du travail dans le secteur du tourisme est sous développé. Ce sous. développement apparait dans les salaires qui sont plus bas que la moyenne des salaires de la population active; il correspond surtout à des conditions de vie au travail très difficiles lau niveau des tâches effectuees et des heures de travaill. Ce sous. developpement se situe aussi au niveau de l'utilisation des ressources humaines de ce secteur: faiblesse dans les modes de sélec. tion, de formation et de promotion du personnel de cette industrie.

Ce sous-développernent a pour corollaire une professionnalisation réduite et cette faible professionnalisation est un obstacle important au développement de l'industrie touristique. Le peu de specialisation des travailleurs de cette industrie rend difficile la définition d'un domaine propre, spécifique au tourisme et. en mẹme temps. ce manque de specialisation empecche les travailleurs de ce secteur de preserver, en tout ou en partie, leur propre domaine d'activite. Pour les travailleurs du tourisme, il est difficile, dans ces conditions, de promouvoir leur competence et leur savoir-faire.

Le sous-developpement des ressources humaines et la quasi-absence de specialisa tion en tourisme sont les résultats d'un trop faible investissement financier dans ces res. sources humaines. Le tourisme n'est pas fait que d'edifices et d'equipements: le développement de cette industrie axee sur "les ser" vices a la personne" va dépendre, en très grande partie, de l'intention et de la capa cité d'investir dans la formation et la professionnalisation des travailleurs en tourisme. En retardant la spécialisation des travailleurs en tourisme et en limitant l'accès de ceux qui ont dejà une formation dans ce domaine (cegeps, I.T.H.Q., universites), on hypothe que lourdement l'avenir de l'industrie touristique du Québec et sa possibilité d'être concurrentielle.
Modele prospectif d'analyse

du marché du travail en tourisme

On peut schematiser ce modèle prospectif

d'analyse par le tableau suivant:

\section{TENDANCE LOURDE}

L'industrie du tourisme est sous-developpee en non specialisee

\section{SOUS.TENDANCES}

- Bas salaires

- Longues heures de travail

- Modes de selection de formation el de promotion peu elaborés

ELEMENTS STRUCTURANTS

- Secteur tertiaire a taible motricite

- Axé essentiellement sur les services a la personne

FAITS PROSPECTIFS DU PRESENT

- Conjoncture economique actuelle favorise la tendance lourde

- Faible investissement dans les ressources humaines dans ce secteur

\section{ÉLEMENTS DESTRUCTURANTS}

- Le seul element pouvant reduire cette ter dance lourde, c'est le désir de professionnali sation des travailleurs de ce secteur

- L'existence de cours aux niveaux collegial et universitaire orientes vers la professionnalisation

\section{FAIT PORTEUR D'AVENIR}

Tendance lourde demeurera importante dans le court terme et le moven terme.

La tendance lourde est l'évolution la plus probable du marche du travail en tourisme au Québec dans le court terme et le moven terme. L'industrie touristique demeurera un secteur de travail peu specialise et sous développé. Cette tendance est déterminee en fonction des donnees du passé portant sur le marché du travail dans ce domaine.

Les sous-tendances de cette tendance lourde résument les effets spécifiques de celle-ci. Ces principaux effets sont plus particulièrement:

- les bas salaires payes dans ce secteur:

- les longues heures de travail:
- les modes rudimentaires de sélection, de formation et de promotion des employés qui travaillent dans ce domaine.

Les éléments structurants regroupent les variables qui vont contribuer au renforcement de la tendance lourde dans le court terme et le moyen terme. La faible motricite ${ }^{111}$ de ce secteur du tertiaire va favoriser le maintien de la tendance lourde. Le deuxieme élement structurant réside dans le caractère relationnel et des services à la personne qui est propre à l'industrie touristique dans son ensemble; ce caractère a peu de chance d'être modifie par les innovations technologiques.

Les faits prospectifs du présent sont des elements negatifs ou positifs pouvant favoriser ou nuire à l'évolution de la tendance lourde. On peut penser que la conjoncture économique actuelle va aller dans le sens de la tendance lourde (faible specialisatoin du secteur du tourismel. La crise economique va reduire les revenus disponibles des particuliers et limiter les depenses des consommateurs dans le secteur du tourisme, ce qui va accentuer le caractère résiduel de cette activité (les vacances).

Certe mauvaise conjoncture va aussi avoir pour effet de limiter les investissements dans les ressources humaines de ce secteur et contribuer ainsi au sous-développement de son marche du travail. La crise economique va aussi accroitre la dependance du domaine touristique vis-aे-vis les mouvements saisonniers et les mouvements alèatoires. Dans l'ensemble les faits prospectifs du présent vont soutenir la tendance lourde definie plus haut!

Les éléments déstructurants sont des variables pouvant provoquer une rupture dans l'evolution d'une tendance lourde. Le seul element destructurant c'est le désir des travailleurs et des cadres de ce secteur de se "professionnaliser", c'est-à dire d'acquérir 
des compétences et des connaissances dans la gestion et l'intervention dans le secteur du tourisme. Ces connaissances pourraient être acquises par le biais des institutions deja existantes (des cours en tourisme sont donnes au niveau collégial et au niveau universitaire).

La professionnalisation de certains travailleurs en tourisme (par le biais des cours ins titutionnels ou autrement) aura, probablement, des effets d'entrainement positifs sur les conditions de travail dans cette indus. trie. Etant mieux formés et étant rassures su leur compétence, ces travailleurs pourraient négocier de meilleures conditions de vie au travail et favoriser ainsi une meilleure ges tion des ressources humaines. Malheureusement ces éléments destructurants ne pourront vraiment agir qu'à très long terme

On peut raisonnablement croire qu'à court terme et à moven terme lune douzaine d'annees) le principal fait porteur d'avenir apparaitra dans la stabilisation de la tendance lourde Isous-spécialisation et sous. developpement du marché du travail dans le secteur du tourisme au Québecl. Le modele prospectif d'analyse du marche du travail en tourisme au Québec pourrait se lire à l'aide d'une simple équation:

$\mathrm{TL}+\mathrm{ST}+\mathrm{ES}+\mathrm{FPP}-\mathrm{ED}=\mathrm{FPA}$

Ainsi, dans cette transposition simplifiee du modèle prospectif, à la tendance lourde

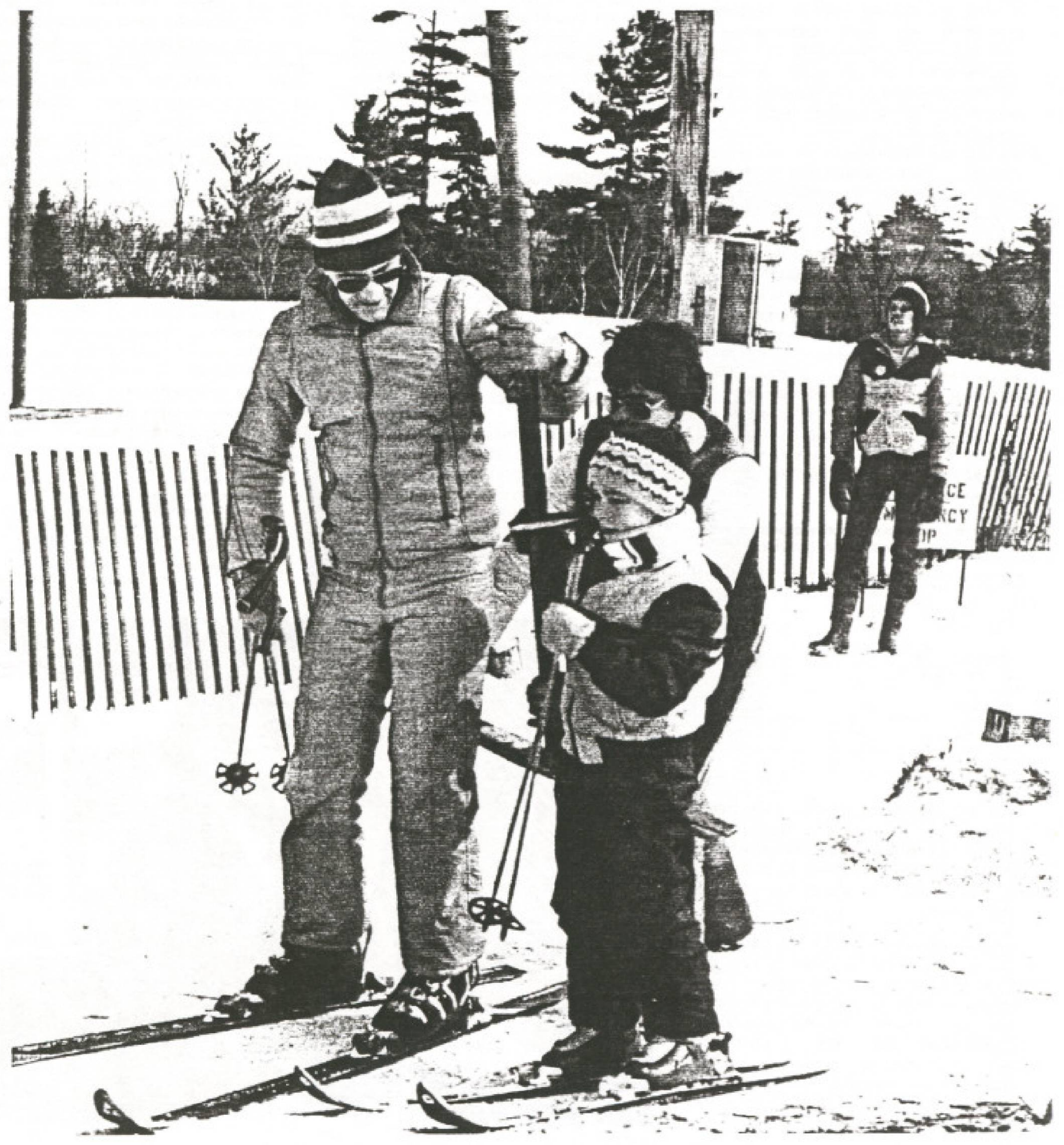


s'additionnent les effets particuliers de certe tendance; les telements structurants sont des invariants qui vont aftermir, dans l'avenir, l'évolution deja forte de cette tendance lourde. Le sous-développement de ce marché du travail sera accentué par les faits prospectifs du présent; le principal tait pros. pectif du présent apparait dans la piétre per formance de notre économie. Cette pitoya. ble conjoncture va consolider la tendance lourde et maximaliser ses principaux effets.

Les elements déstructurants sont, ici, néga tifs: ils vont réduire la vitesse de certe tendance lourde et attenuer un peu ses princi paux effets. Ces élements déstructurants ne font "pas le poids" face à la force d'inertie de la tendance lourde; ils n'auront un impact important que dans plusieurs années. Ce qui découle de notre analyse, en terme de fait porteur d'avenir pour le marché du travail en tourisme au Québec, c'est que les ressources humaines resteront sous-utilisées, que la tendance lourde se maintiendra sans difficulte importante!

Des hypothèses sur l'avenir du marche du travail en tourisme au Québec En tenant compte de la force de la tendance lourde, il est certain que celle-ci sera prépon dérante dans les prochaines années. A plus long terme, d'autres hypotheses sont possibles; ces hypothèses se greffent, d"une part sur le degre de professionnalisation des travailleurs de ce secteur et, d'autre part, sur la croissance de ce marché au plan de la création d'emplois nouveaux; en fonction de ces éléments on peut résumer dans un tableau ces hypothèses:

Degré de professionnalisation/croissance des emplois dans le secteur du tourisme

\begin{tabular}{|c|c|c|}
\hline $\begin{array}{l}\text { Degre de } \\
\text { croissance } \\
\text { du marche } \\
\text { du travail }\end{array}$ & Faible & \\
\hline Faible & Fort \\
\hline Fort & 1 & \\
\hline
\end{tabular}

Dans l'hypothèse 1 la tendance lourde est amplifiée par la lente crise economique actuelle: à une faible professionnalistation correspond une faible croissance du marché des emplois, c'est la situation actuelle qui se perpetue.

Dans la deuxième hypothese on assiste a une reprise économique importante qui aurait pour principale consequence une croissance forte de la demande de travailleurs pour cette industrie. Au niveau de la specialisation, celle-ci resterait faible donc peu d'implication et de creativite: les bas salaires et la mauvaise condition de travail demeureraient le lot de la plupart des trá vailleurs de ce secteur.
La troisieme hypothese suppose un niveau élevé de professionnalisation couplè ầ une faible croissance du marche du travail. Avec cette hypothese on peut penser que "les canards boiteux", les elements les moins "performants", auront ete elimines par la crise favorisant ainsi la specialisation des tra vailleurs de ce secteur (pour lutter contre la concurrence, les entreprises devront se spé cialiser â outrance et investir dans les ressources humainesl.

La quatrième hypothèse présume une forte croissance du nombre des emplois dans cette industrie ainsi qu' une forte professionnalisation; la jonction de ces deux croissances provoquerait la naissance d'une véritable industrie du tourisme axee sur le caractere professionnel et de bonnes conditions de travail.

\section{Du sous-développement à la} professionnalisation

Ces hypotheses permettent d' allier la retros pective et la prospective afin de donner une image claire de l'etat du marché du travail en tourisme au Québec dans les vingts prochaines années. Les deux premières hypotheses ont relativement peu de chance de se realiser dans le futur car elles correspondent respectivement au decollage de l'indus trie touristique (avant 1960) et au tourisme de masse des années 1960-1975.

La troisième hypothèse implique une rupci l'industrie touristique repartirait sur de nouvelles bases en misant fortement sur le developpement des ressources humaines et en valorisant la planification, l'innovation et Ianimation. On assisterait, de cette façon, à une nouvelle structuration du champ du tourisme, à une grande concertation entre ture entre l'avant et l'après crise; après celle-

les intervenants et, enfin, à une plus grande integration à l'ensemble économiqué

La quatrième hypothèse suppose une forte reprise sur le plan économique; cette forte reprise associé à une forte professionnalisation ferait sûrement du tourisme une industrie de tout premier plan. Cette hypothese est peu probable avant les annees 1995. La longue marche du sous. developpement à la professionnalisation demandera donc encore plus d'une décennie.

La lente règression de la tendance lourde se fera uniquement par le biais de la formation et de la professionnalisation des travailleurs de ce secteur. L'erosion de la tendance décrite au debut de ce texte demandera d'importants investissements au plan intellectuel et au plan financier; il semble aujourd'hui évident qu'une force de travail plus scolarisee et plus spécialisée peut effectuer des tâches plus complexes avec un rendement superieur; ces investissements auraient aussi, pour conséquence immédiate, d'améliorer la qualité de la vie au tra. vail des travailleurs du tourisme.

En investissant dans les ressources humai. nes, I'industrie touristique deviendra plus performante et changera les conditions de travail de ses employé(es). La dégradation de la tendance lourde exigera une remise en cause des attitudes et des comporte ments actuels ainsi qu'une prise de cons. cience du sous-développement du marché du travail $f$

(1) Au suet du "hertiaie moteur" wor

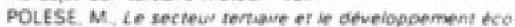
namigue reponal wers un madede operatronnel des actiri

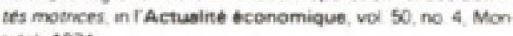
treal. 1974

pour te tertiare vor.

COSSETTE. A. Lo tortiariantion de I'bconomie gutbe boikt, Edtion Gatian Monin Checoutima 198:

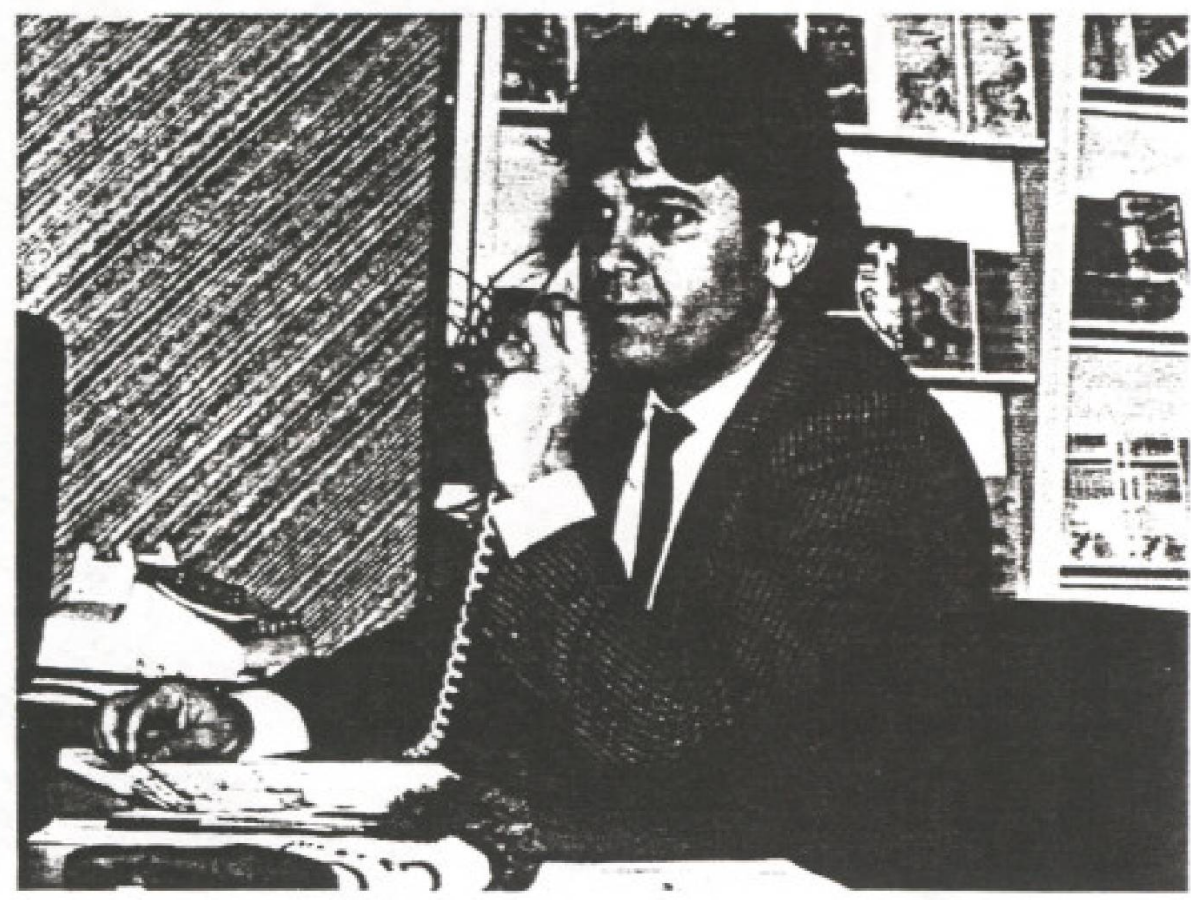

